\title{
Mitochondrial Complex I Deficiency Leads to Increased Production of Superoxide Radicals and Induction of Superoxide Dismutase
}

\author{
Sari Pitkänen‡ and Brian H. Robinson* \\ $*$ Departments of Pediatrics and Biochemistry, University of Toronto, Toronto, Ontario; and ${ }^{\ddagger}$ Department of Genetics, \\ The Research Institute, The Hospital for Sick Children, Toronto, Ontario
}

\begin{abstract}
Mitochondria were isolated from skin fibroblast cultures derived from healthy individuals (controls) and from a group patients with complex I (NADH-CoQ reductase) deficiency of the mitochondrial respiratory chain. The complex I deficient patients included those with fatal infantile lactic acidosis (FILA), cardiomyopathy with cataracts (CC), hepatopathy with tubulopathy (HT), Leigh's disease (LD), cataracts and developmental delay (CD), and lactic acidemia in the neonatal period followed by mild symptoms (MS). Production of superoxide radicals, on addition of $\mathrm{NADH}$, were measured using the luminometric probe lucigenin with isolated fibroblast mitochondrial membranes. Superoxide production rates were highest with CD and decreased in the order $\mathrm{CD}>>\mathrm{MS}>\mathrm{LD}>$ control $>\mathrm{HT}>$ FILA = CC. The quantity of Mn-superoxide dismutase (MnSOD), as measured by ELISA techniques, however, was highest in CC and FILA and lowest in CD. Plots of MnSOD quantity versus superoxide production showed an inverse relationship for most conditions with complex I deficiency. We hypothesize that oxygen radical production is increased when complex I activity is compromised. However, the observed superoxide production rates are modulated by the variant induction of MnSOD which decreases the rates, sometimes below those seen in control fibroblast mitochondria. In turn, we show that the variant induction of MnSOD is most likely a function of the change in the redox state of the cell experienced rather than a result of the complex I defect per se. (J. Clin. Invest. 1996. 98:345-351.) Key words: manganese superoxide dismutase - superoxide radical • complex I deficiency • Leigh's disease • cataracts
\end{abstract}

\section{Introduction}

The mitochondrial respiratory chain produces reactive oxygen species (ROS) ${ }^{1}$ as by-products of electron transport (1). These radicals in the form of either superoxide or hydroxyl radicals are thought to be produced by interaction of molecular oxygen with semi-quinone or -flavone species resulting from transient one electron transfer reactions with the respiratory chain complexes $(2,3)$. The superoxide anion radical $\left(\mathrm{O}_{2}^{-}\right)$appears to be

Address correspondence to Brian $\mathrm{H}$. Robinson, $\mathrm{PhD}$, The Research Institute, The Hospital for Sick Children, 555 University Avenue, Toronto, Ontario, Canada M5G 1X8. Phone: 416-813-5989; FAX: 416-813-4931; E-mail: bhr@resunix.ri.sickkids.on.ca

Received for publication 20 March 1996 and accepted in revised form 9 May 1966.

J. Clin. Invest.

(C) The American Society for Clinical Investigation, Inc.

0021-9738/96/07/0345/07 \$2.00

Volume 98, Number 2, July 1996, 345-351 the first oxygen reduction product generated by mitochondria under both physiological and pathological conditions (1). Superoxide is removed by superoxide dismutase to hydrogen peroxide. Hydrogen peroxide in turn, under certain circumstances, may react with free iron or copper to produce the more damaging hydroxyl radical (3). There are two intracellular dismutase enzymes: the manganese superoxide dismutase (MnSOD) located intramitochondrially, and the copper-zinc superoxide dismutase (CuZnSOD) located in cytosol (3). The expression of the MnSOD enzyme unlike the CuZnSOD enzyme undergoes considerable modulation, being upregulated by cytokines (4) or by hyperoxia (5).

Superoxide production from sites within the respiratory chain has been measured in isolated mitochondria and mitochondrial membranes, and various sites of production within the respiratory chain have been proposed in complex I (NADH-CoQ reductase) and in complex III (ubiquinol-cytochrome $c$ reductase) (3). Little information is available on oxygen radical production in genetically determined respiratory chain defects, especially in complex I deficiency. In this respect we recently discovered evidence of excessive reactive oxygen radical production in a family with cardiomyopathy, cataracts, and complex I deficiency of the mitochondrial respiratory chain (6). Here we report that superoxide production in skin fibroblast mitochondria from a spectrum of patients with complex I deficiency can be elevated or decreased depending on the state of MnSOD induction. The reasons for these differences were investigated.

\section{Materials and Methods}

Patients. We made a detailed study of 13 patients with functional complex I deficiency in cultured skin fibroblasts. Patients were sorted into six different categories according to clinical history:

(1) Fatal Infantile Lactic Acidosis (FILA)

(2 patients)

(2) Leigh's Disease (LD)

(3) Cardiomyopathy with Cataracts (CC)

(2 patients)

(4) Hepatomegaly with Renal Tubulopathy (HT)

(2 patients)

(5) Cataracts and Developmental delay (CD)

(2 patients)

6) Lactic acidemia in the neonatal period followed by Mild Symptoms (MS)

(2 patients)

(3 patients)

Some further patient fibroblast samples were also employed from patients with various defects in complexes I, III, IV, and the pyruvate dehydrogenase complex to facilitate the correlation of expression of MnSOD and alterations in redox state.

1. Abbreviations used in this paper: $\mathrm{CC}$, cardiomyopathy with cataracts; $\mathrm{CD}$, cataracts with developmental delay; CuZnSOD, copperzinc superoxide dismutase; FILA, fatal infantile lactic acidosis; HT, hepatomegaly with renal tubulopathy; LD, Leigh's disease; L/P, lactate to pyruvate; MnSOD, manganese superoxide dismutase; MS, mild symptoms; ROS, reactive oxygen species. 
Cell culture and enzymatic assays. Human skin fibroblasts were grown from explants of forearm skin biopsy tissue (taken after informed consent). Culture medium was Eagles alpha-minimal essential medium supplemented with $10 \%$ fetal calf serum and $10.5 \mathrm{mM}$ glucose, and $1 \mathrm{mM}$ uridine to sustain normal growth rates. Two to three different skin fibroblast cell lines cultured from biopsies of normal children were used as controls.

Lactate to pyruvate ratios were determined as described previously (7) by measuring the levels of lactate and pyruvate after an exposure of $1 \mathrm{~h}$ to $1 \mathrm{mM}$ glucose in Krebs phosphate buffer. Mitochondria were isolated from cultured skin fibroblasts (8) and the activity of the rotenone-sensitive NADH-cytochrome $c$ reductase (complexes I + III) was measured by the method of Moreadith et al. (9). Activities of the other respiratory chain components were measured from whole cell extracts as follows: cytochrome oxidase (complex IV) by the method of DiMauro et al. (10), and succinate cytochrome $c$ reductase by following the reduction of cytochrome $c$ (complexes II + III) by the method of Fischer et al. (11).

Standardization of the luminescent superoxide assay. Superoxide was generated by enzymatic reaction of xanthine oxidase on its substrate hypoxanthine and estimated by two different methods with minor changes $(12,13)$. Spectrophotometric (12) and luminometric methods (13) were used in order to compare the results in two different systems and convert the luminometer units (counts/s) to $\mathrm{nmol} / \mathrm{min} /$ $\mathrm{mg}$ of mitochondrial protein in skin fibroblast mitochondrial assays.

Stock solutions for spectrophotometric assays were prepared as follows: Hypoxanthine (Sigma Chemical Co., St. Louis, MO) was 50 $\mathrm{mM}$ in $500 \mathrm{mM} \mathrm{KOH}$ which was used within $1 \mathrm{wk}$. Xanthine oxidase (type III from buttermilk; Sigma Chemical Co.) $0.5 \mathrm{U} / \mathrm{ml}$ was prepared in experimental buffer $\left(50 \mathrm{mM} \mathrm{KH}_{2} \mathrm{PO}_{4} \times \mathrm{HPO}_{4}\right.$ with $1 \mathrm{mM}$ EDTA at $\mathrm{pH}$ 7.4). Horse heart ferricytochrome $c$ (type VI; Sigma Chemical Co.) $1 \mathrm{mM}$ was prepared in $\mathrm{H}_{2} \mathrm{O}$. The assay system consisted of $1 \mathrm{ml}$ experimental buffer, $25 \mu \mathrm{M}$ ferricytochrome $c, 500 \mu \mathrm{M}$ hypoxanthine in both cuvettes in a split beam spectrophotometer
(Pye-Unicam SP1800). After the base line was established, xanthine oxidase was added from 0.5 to $5.0 \mathrm{U} / 1$ to one cuvette and reduction of ferricytochrome $c$ was followed at $550 \mathrm{~nm}$ at $25^{\circ} \mathrm{C}$, compared to a control cuvette with no xanthine oxidase.

For the luminometric determination a chemiluminescent probe of high quantum efficiency, lucigenin (10,10'-dimethyl-9,9'-biacridinium dinitrate; Sigma Chemical Co.) was used to quantify superoxide production monitored as emitted light by a sensitive luminometer (luminometer model 1253; Bio-Orbit, Oy, Finland). The concentration of the working solution of lucigenin was assayed spectrophotometrically using an extinction coefficient at $410 \mathrm{~nm}$ of $8,900 \mathrm{M}^{-1} \mathrm{~cm}^{-1}$. The assay system consisted of the experimental buffer, $20 \mu \mathrm{M}$ lucigenin, and $500 \mu \mathrm{M}$ hypoxanthine. Xanthine oxidase (from 0.5 to $5.0 \mathrm{U} / \mathrm{l}$ ) was added to initiate oxidation of hypoxanthine. The luminometer was set to count for 20 intervals of 1 -s each every fifth second and the temperature was $25^{\circ} \mathrm{C}$.

Determination of superoxide production in isolated mitochondria. Lucigenin as a chemiluminescent probe was employed to measure superoxide production from the respiratory chain. $15 \mu \mathrm{g}$ of frozen-thawed mitochondria isolated from fibroblast cultures was added to a solution of $10 \mathrm{mM} \mathrm{K}_{2} \mathrm{HPO}_{4}, \mathrm{pH} 10.5$, and the cuvette was placed into the counter. Background was subtracted and counting was initiated. $\beta$-Nicotinamide adenine dinucleotide (NADH, $50 \mu \mathrm{g}$ in $10 \mu \mathrm{l}$ $\mathrm{H}_{2} \mathrm{O}$ ) was added into the cuvette as the substrate initiating respiratory chain electron transport. NADH also functioned as a reducing agent via diaphorase in fibroblast mitochondria preparations for the lucigenin probe which is a prerequisite for the reaction of lucigenin with superoxide (14). Rates of superoxide production were expressed either as counts per second measured at 5-s intervals or were converted using a standard curve to $\mathrm{nmol} / \mathrm{min} / \mathrm{mg}$ mitochondrial protein from the counts/s at $30 \mathrm{~s}$ (steady state) after starting the reaction.

Manganese superoxide dismutase measurements by ELISA. The MnSOD ELISA kit (Bender MedSystems, Vienna, Austria), an enzyme-linked immunosorbent assay, was used to quantify MnSOD lev-

Table I. Lactate to Pyruvate Ratio, NADH-Cytochrome c Reductase Activity, Superoxide Production, and MnSOD Quantity Measured in Cultured Skin Fibroblasts or in Isolated Mitochondria

\begin{tabular}{|c|c|c|c|c|}
\hline Patient & $\mathrm{L} / \mathrm{P}$ ratio & $\begin{array}{c}\text { NADH-cytochrome } \\
c \text { reductase }\end{array}$ & $\begin{array}{l}\text { Superoxide } \\
\text { (steady state) }\end{array}$ & MnSOD \\
\hline & & $\mathrm{nmol} / \mathrm{min} / \mathrm{mg}$ & $\mathrm{nmol} / \mathrm{min} / \mathrm{mg}$ & $n g / m g$ prot \\
\hline C 4212 & $26.3 \pm 3.5(12)$ & $133 \pm 8.4(8)$ & $5.67 \pm 0.4(4)$ & $90.8 \pm 8.4(5)$ \\
\hline C 3639 & $30.4 \pm 4.2(11)$ & $125 \pm 10.9(8)$ & $5.28 \pm 0.3(2)$ & $85.7 \pm 11.9(3)$ \\
\hline C $3639+$ Rotenone $1 \mu \mathrm{M}$ & $347 \pm 26(5)^{*}$ & $0.00 \pm 0.0(5)^{*}$ & $7.14 \pm 0.6(2)$ & $125 \pm 22(2)$ \\
\hline MS 4231 & $69.0 \pm 8.6(13)^{*}$ & $73.7 \pm 6.3(3)^{*}$ & $7.96 \pm 0.8(3)^{*}$ & $174.7 \pm 18.6(3)^{*}$ \\
\hline MS 5716 & $34.3 \pm 4.1(3)$ & $94.4 \pm 8.8(7)^{*}$ & $9.81 \pm 0.2(2)^{*}$ & $79.5 \pm 16.1(3)$ \\
\hline MS 5066 & $55.9 \pm 9.2(9)^{*}$ & $85.6 \pm 7.3(5)^{*}$ & $9.67 \pm 1.2(2)^{*}$ & $95.0 \pm 6.6(3)$ \\
\hline CD 7123 & $15.4 \pm 0.8(4)$ & $43.6 \pm 3.3(16)^{*}$ & $14.4 \pm 2.1(2)^{*}$ & $105.5 \pm 14.9(4)$ \\
\hline CD 7133 & $15.7 \pm 2.1(4)$ & $53.7 \pm 7.3(11)^{*}$ & $11.9 \pm 0.8(3)^{*}$ & $94.7 \pm 17.2(5)$ \\
\hline LD 3690 & $67.8 \pm 13.3(8) *$ & $47.7 \pm 8.2(7)^{*}$ & $6.97 \pm 0.4(2)$ & $92.6 \pm 6.1(5)$ \\
\hline LD 5509 & $50.4 \pm 3.6(4)^{*}$ & $36.4 \pm 6.5(7)^{*}$ & $6.57 \pm 0.2(3)$ & $82.0 \pm 5.4(4)$ \\
\hline CC 5221 & $101.9 \pm 26.1(6)^{*}$ & $75.3 \pm 6.0(14)^{*}$ & $2.58 \pm 0.0(2)^{*}$ & $336.5 \pm 79.3(5) *$ \\
\hline CC 5219 & $63.5 \pm 10.1(4)^{*}$ & $63.5 \pm 10.1(4)^{*}$ & $4.16 \pm 0.2(5)^{*}$ & $244.8 \pm 59.9(3) *$ \\
\hline HT 5624 & $93.1 \pm 21.7(5)^{*}$ & $26.7 \pm 3.2(3)^{*}$ & $4.72 \pm 0.6(2)$ & $234.9 \pm 21.0(3)^{*}$ \\
\hline HT 6275 & $135.3 \pm 14.9(6)^{*}$ & $40.6 \pm 6.9(5)^{*}$ & $4.96 \pm 0.2(3)$ & $227.1 \pm 17.5(3) *$ \\
\hline FILA 4091 & $169.0 \pm 68.0(4)^{*}$ & $47.6 \pm 4.6(5) *$ & $3.29 \pm 0.1(2)^{*}$ & $229.7 \pm 63.3(5)^{*}$ \\
\hline FILA 5340 & $221.7 \pm 67.6(5) *$ & $37.6 \pm 3.7(7) *$ & $4.40 \pm 0.3(2)$ & $233.0 \pm 22.5(3) *$ \\
\hline
\end{tabular}

Superoxide rates were calculated from the production curves (Fig. 5). Measurements for NADH-cytochrome $c$ reductase, superoxide production rate, and MnSOD by ELISA are given as mean \pm SEM for a number of different mitochondrial preparations given in parentheses. Lactate to pyruvate $(\mathrm{L} / \mathrm{P})$ ratios determined in whole cell cultures are given as the mean \pm SEM for a number of determinations given in parentheses. FILA, Fatal infantile lactic acidosis; $C C$, cardiomyopathy with cataracts; $H T$, hepatomegaly with tubulopathy; $L D$, Leigh's disease; $C D$, cataracts and developmental delay; $M S$, lactic acidemia in the neonatal period followed by mild symptoms; $C$, controls. *Indicates a significant difference compared with control values at level of $P=<0.05$ or less. 
els in frozen-thawed mitochondrial samples made from patient's cultured fibroblasts. Each sample was measured in duplicate for a number of different mitochondrial preparations given in parentheses.

Northern blot analysis. Northern blotting was carried out using total RNA prepared from fibroblasts as described previously (15) and electrophoresed in $1 \%(\mathrm{wt} / \mathrm{vol})$ agarose containing $1.8 \%(\mathrm{wt} / \mathrm{vol})$ formaldehyde. The RNA was transferred to a Hybond- ${ }^{+}$support membrane (Amersham Corp., Arlington Heights, IL). This was then incubated with a ${ }^{32} \mathrm{P}$-labeled cDNA probe obtained by random priming a MnSOD cDNA template followed by washing in standard buffers (15). Total RNA loading was monitored by simultaneously probing on the same blot for mitochondrial EFTU (mitochondrial translation factor, $1.7 \mathrm{~kb}$ ) (Merante, F., M. Ling, H.-S. Chen, and B.H. Robinson. 1995. manuscript submitted).

Statistical analysis. Linear regression was used to compare residual activities of complexes I and III (NADH cytochrome $c$ reductase) and lactate to pyruvate ratios or MnSOD and lactate to pyruvate ratios.

\section{Results}

Cultured skin fibroblast investigations. A series of patients with MS, LD, CC, HT, and FILA were investigated because the lactate to pyruvate ratio observed in cultured skin fibroblasts was high, indicating a respiratory chain defect (Table I). Further analyses were done to investigate the cause of lactic acidemia and the activities of rotenone-sensitive NADH-cytochrome $c$ reductase (complexes I + III) was found to be decreased in all patients (Table I). The CD patients who had normal lactate to pyruvate ratios in fibroblasts were known to have complex I defects from analysis of muscle biopsies. The activities of rotenone-sensitive NADH-cytochrome $c$ reductase in patients varied between $20-73 \%$ of controls for different patient's fibroblast mitochondria. Cyochrome oxidase (complex IV) and succinate-cytochrome $c$ reductase (complexes II and III) were always normal, or in some cases slightly elevated. This pattern is used to establish the diagnosis of complex I deficiency $(7,8)$. All of these patients had mild or severe lacticacidemia with normal activities of both pyruvate carboxylase and the pyruvate dehydrogenase com-

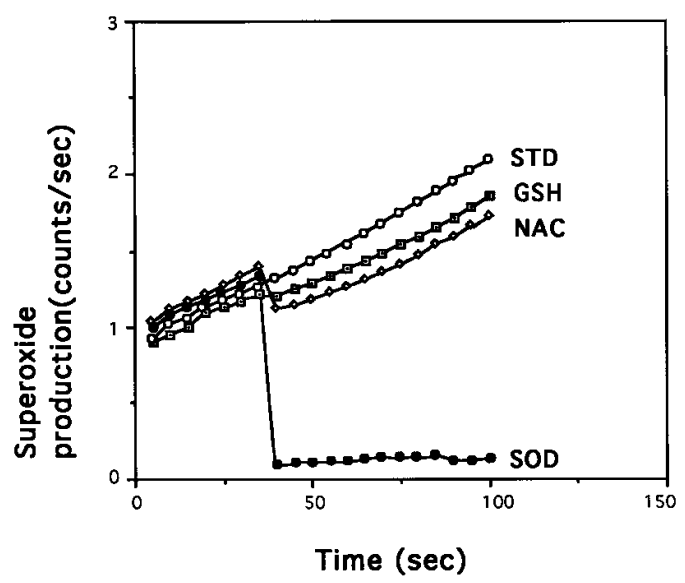

Figure 1. Superoxide dismutase affects lucigenin luminescence. In the assay system containing $15 \mu \mathrm{g}$ rat heart mitochondria in $1 \mathrm{ml}, 20$ $\mu \mathrm{M}$ lucigenin was added to $50 \mu \mathrm{g}$ NADH to initiate the respiratory chain electron transport. After $35 \mathrm{~s} 150 \mu \mathrm{g}$ reduced glutathione ( $G S H /$ final concentration $0.46 \mathrm{mM})(\square)$; $0.02 \mathrm{U}$ copper/zinc-superoxide dismutase $(S O D)(\bullet) ; 80 \mu \mathrm{g} \mathrm{N}$-acetyl cysteine (NAC/final concentration $0.46 \mathrm{mM}$ ) was added) $(\diamond)$. STD indicates standard curve with no additions $(\bigcirc)$.

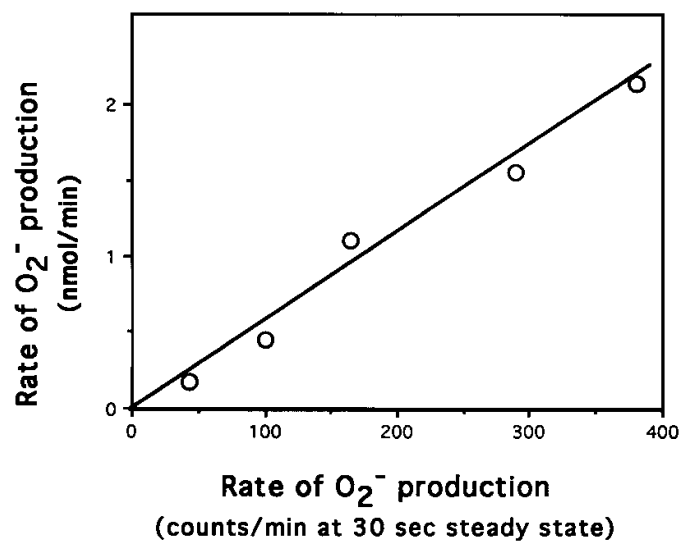

Figure 2. Correlation of superoxide generation by luminometry and spectrophotometry. Superoxide was generated by enzymatic reaction of xanthine oxidase on its substrate hypoxanthine and estimated by spectrophotometric and luminometric methods. Using the same concentrations of substrates the production of superoxide could be converted from luminometric units counts/min (counted at steady state at $30 \mathrm{~s}$ ) to spectrophotometric units $\mathrm{nmol} / \mathrm{min}$.

plex in skin fibroblasts. Corroborating results have been described by us previously (Pitkänen, S., A. Feigenbaum, R. Laframboise, and B.H. Robinson. 1996. manuscript in press.) to demonstrate this wide clinical spectrum of complex I deficiency. Isolated complex I deficiency is a seldom reported clinical defect with most published work referring to patients with FILA or LD $(8,9,16)$.

Establishment of a sensitive superoxide assay system. Detection of superoxide production by standard spectrophotometric methods was not feasible because with the small amounts of fibroblast mitochondria ( $0.4 \mathrm{mg}$ protein per preparation) available the rates would be barely detectable. To overcome this problem a sensitive luminometric assay for superoxide was established and calibrated with respect to the standard spectrophotometric assay.

Oxygen radicals were produced by enzymatic reaction of xanthine oxidase on its substrate hypoxanthine and rates of production were estimated by both luminometer and spectrophotometer. Lucigenin, in its reduced state, undergoes a rapid light-producing reaction with the superoxide radical under the conditions used in this assay system (14). To demonstrate that the assay system displays superoxide radical generation, different free radical scavengers were added into the assay system. By adding excess superoxide dismutase (which specifically removes superoxide) the production of superoxide was reduced by $92 \%$. On the other hand, hydrogen peroxide scavengers such as $N$-acetyl cysteine or glutathione did not have a great effect on superoxide production (Fig. 1). Determination of superoxide production by both spectrophotometry and luminometry with subsequent use of the standard curve generated (Fig. 2) allowed us to describe superoxide production in $\mathrm{nmol} / \mathrm{min}^{-1} / \mathrm{mg}^{-1}$ of mitochondrial protein.

Superoxide production from mitochondria. Mitochondria disrupted by freeze-thawing were incubated with the luminometric probe lucigenin to assess the production of superoxide from the respiratory chain on addition of NADH. When patient's fibroblast mitochondria were tested, those from the patient categories CD, LD, and MS showed increased superoxide production compared to controls, while those patients 


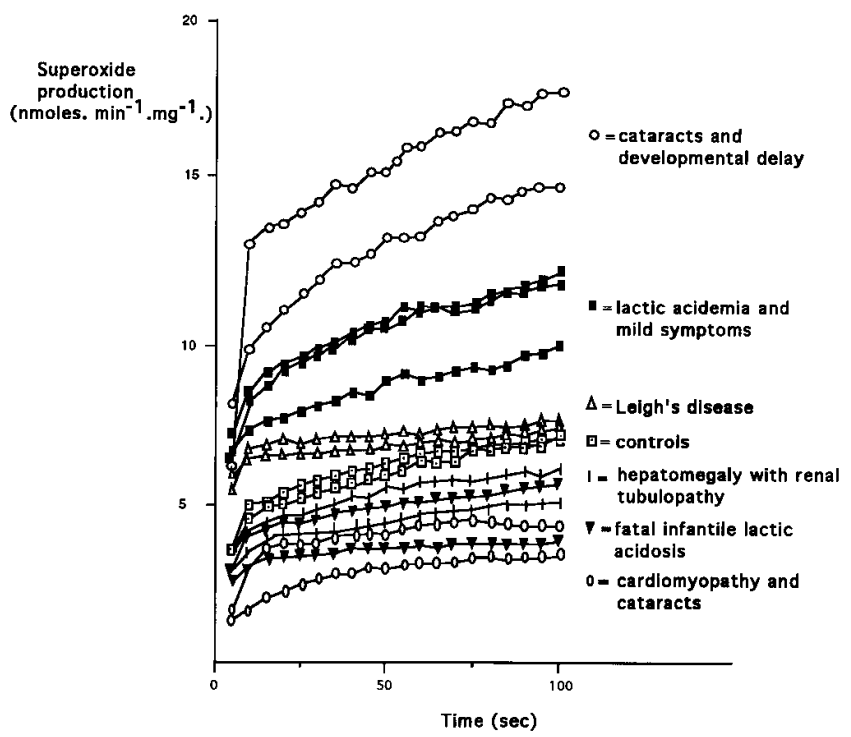

Figure 3. Time course of lucigenin-dependent chemiluminescence catalyzed by mitochondria isolated from skin fibroblast cultures. Measurements were carried out as described in Materials and Methods using $15 \mu \mathrm{g}$ of mitochondrial protein. The steady state of superoxide production decreased in the order: cataracts and developmental delay $>>$ mild symptoms $>$ Leigh's disease $>$ control $>$ hepatomegaly with renal tubulopathy $>$ fatal infantile lactic acidoses $=$ cardiomyopathy with cataracts. Each measurement was repeated at least three times using three different mitochondrial preparations from each cell line. Mean rates of superoxide production \pm SEM are given in Table I.

from categories HT, FILA, and CC showed decreased rates relative to controls (Fig. 3). Superoxide production was increased in normal mitochondria by addition of the respiratory chain inhibitors rotenone (complex I inhibitor) and antimycin (complex III inhibitor) (Fig. 4). Addition of rotenone or antimycin to patient and control mitochondria caused similar increments in superoxide production (data not shown). Addi-

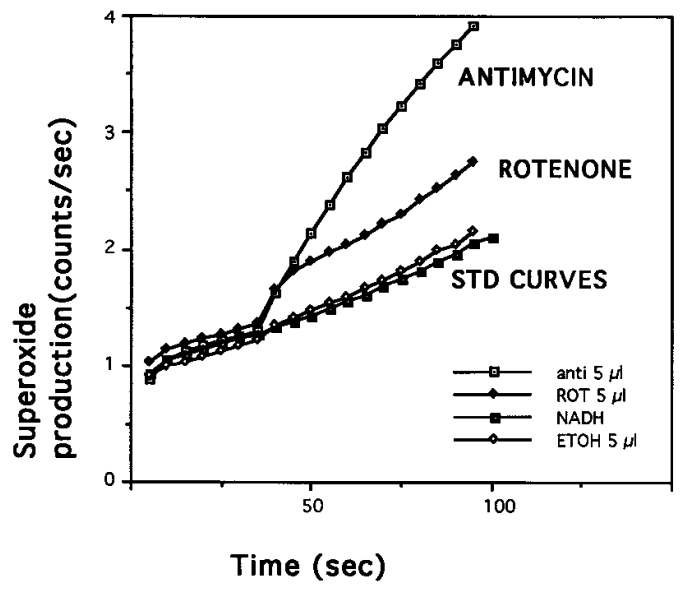

Figure 4. Effect of rotenone and antimycin on mitochondrial superoxide production. To the assay system containing $15 \mu \mathrm{g}$ rat heart mitochondria in $1 \mathrm{ml}$ buffer with $20 \mu \mathrm{M}$ lucigenin the reaction was started by addition of $50 \mu \mathrm{g}$ NADH. After $35 \mathrm{~s}$ rotenone (final concentration $10 \mu \mathrm{M})(\diamond)$ or antimycin (final concentration $100 \mu \mathrm{M})(\square)$ was added. tion of NADPH did not elicit superoxide production in mitochondrial preparations.

Manganese superoxide dismutase measured by MnSOD ELISA. Accurate determinations of MnSOD protein in mitochondrial samples by an ELISA technique showed that MnSOD levels in control fibroblast lines were 85.7-90.8 ng/ $\mathrm{mg}$ of mitochondrial protein. Complex I deficient fibroblast lines displayed values from the normal range up to $336.5 \mathrm{ng} /$ $\mathrm{mg}$ of mitochondrial protein. Patients with complex I deficiency who had CC, FILA, and HT had 2.6-3.8-fold higher values compared to controls. The patients with $\mathrm{LD}, \mathrm{CD}$, or mild symptoms had wild-type or marginally elevated levels compared to control values (Table I).

In general, fibroblast lines showing increased levels of MnSOD to the greatest extent seemed to be those with the biggest change in redox state determined by lactate to pyruvate ratio (Fig. 5). MnSOD ELISA measurements were then made on a much larger series of patient fibroblast mitochondria accumulated over the past ten years from patients with defects in energy metabolism. These mitochondria had been stored at $-70^{\circ} \mathrm{C}$ and though some enzyme activities had partially decayed over this period, they were suitable for immunological studies such as this one. The stored $\left(-70^{\circ} \mathrm{C}\right)$ isolated mitochondria from this collection were thawed, the protein content determined, and duplicate $15 \mu \mathrm{g}$ protein aliquots were used in the ELISA assay for MnSOD. These samples were indistinguishable from those mitochondria freshly prepared from fibroblasts except for the length of time in storage at $-70^{\circ} \mathrm{C}$. The amount of MnSOD protein plotted against the lactate to pyruvate ratio of the complex I deficiency patient fibroblast lines showed a compelling relationship between the two parameters $\left(P<0.02, \mathrm{R}^{2}=0.62, n=23\right)$ (Fig. 5). For patient fibroblast mitochondria with deficiencies of pyruvate carboxylase, pyruvate dehydrogenase, complex III, or complex IV, values for MnSOD did not increase with $\mathrm{L} / \mathrm{P}$ ratio (Fig. 5). Two patients, however, with complex IV deficiency did show a significant increment in MnSOD relative to the increase in L/P ratio compared to the other controls. Addition of the respiratory chain inhibitor rotenone to cells $72 \mathrm{~h}$ before

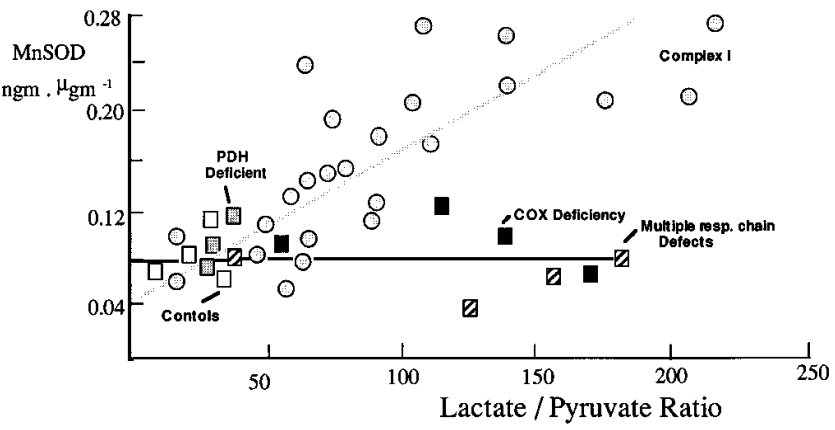

Figure 5. Correlation of the level of MnSOD and the $\mathrm{L} / \mathrm{P}$ ratio in fibroblasts for complex I deficiency. The quantity of MnSOD measured by ELISA in fibroblast mitochondria is plotted as a function of the lactate to pyruvate ratio in skin fibroblast cultures. For patient cell lines with complex I deficiency, there is significant correlation (ㅇ). For patient fibroblast mitochondria with pyruvate dehydrogenase (周), complex III and multiple respiratory chain defects $(\mathbb{Z})$, or complex IV deficiency ( $\square)$ there was no such correlation. See text for details of statistical analysis. Controls are designated ( $\square$ ). 


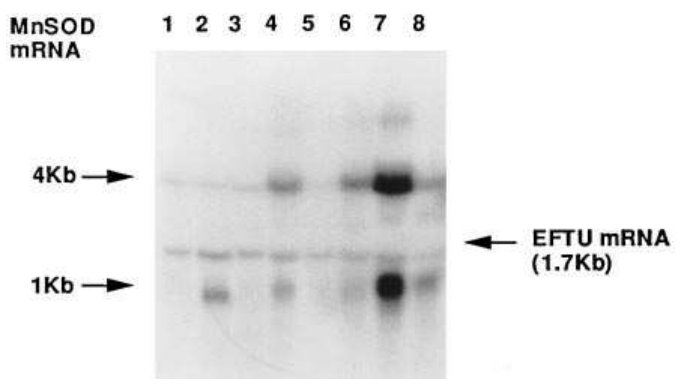

Figure 6. Northern blot analysis. Skin fibroblast total RNA was probed with ${ }^{32} \mathrm{P}$-labeled probes for MnSOD and mitochondrial EFTU (mitochondrial elongation factor Tu, $1.1 \mathrm{~kb}$ ). Lane 1, control; lanes 2 and 3, patients with hepatomegaly and renal tubulopathy; lanes 4 and 6 , patients with fatal infantile lactic acidosis; lane 5 , patient with Leigh's disease; lanes 7 and 8 , patients with cardiomyopathy and cataracts. MnSOD has two mRNA species, at 1 and $4 \mathrm{~kb}$, while the EFTU mRNA is at $1.7 \mathrm{~kb}$.

harvesting and preparation of mitochondria resulted in a small $(20 \%)$ increase in MnSOD (Table I).

Northern blot analysis. Northern blot analysis using a cDNA probe for MnSOD showed that the mRNA levels in fibroblasts were increased in proportion to the increased levels of MnSOD protein detected by ELISA. MnSOD message was markedly increased in five out of 13 patients in the following clinical groups: cardiomyopathy with cataracts (both patients) $>$ fatal infantile lactic acidosis (both patients) $>$ hepatomegaly with renal tubulopathy (one patient) (Fig. 6).

\section{Discussion}

Rotenone-sensitive NADH-cytochrome $c$ reductase (complexes I + III) activities were measured in frozen thawed mitochondria as the best estimate of the deficit in complex I activity. Direct measurement of NADH-CoQ reductase (Complex I) activity in fibroblast mitochondria is technically unreliable due to the extremely high activity of rotenone-insensitive nonspecific diaphorase present in fibroblast mitochondrial preparations. In our hands measurement of NADH-CoQ reductase in fibroblast mitochondria is not precise enough to detect partial defects as present in cardiomyopathy and cataracts (6) or in patients with lactic acidemia in the neonatal period followed by mild symptoms. The disturbance in the redox state of these fibroblasts was measured by determination of the lactate to pyruvate $(\mathrm{L} / \mathrm{P})$ ratio. The fibroblast lines from the patients with most severe complex I defect involving either FILA or HT showed high L/P ratios indicating a greater disturbance in redox state. There seemed to be a correlation with residual activity in complexes $\mathrm{I}+\mathrm{III}$ and $\mathrm{L} / \mathrm{P}$ ratios in these patients compared to controls. Since patients with fatal $\mathrm{CC}$ had also high $\mathrm{L} / \mathrm{P}$ ratios but only a partial defect in complex I compared to LD and CD patients, we previously hypothesized that free radical involvement in the pathology was at least partially responsible for the severe outcome of this disease (6).

The use of the luminometric probe lucigenin permitted us to examine in a detailed and comparative fashion the rates of superoxide production from fibroblast mitochondria derived from patients with complex I deficiency. Since these patients displayed a variety of phenotypes, we were anxious to see if any of these phenotypes might correlate with excess production of reactive oxygen species. We found considerable variability in the rates of superoxide production compared to controls but nevertheless in general the rate of superoxide production followed clinical symptoms, i.e., the patients with the same symptoms seemed to produce the same amount of superoxide. Superoxide production rates were highest with the patients with $\mathrm{CD}$ and decreased in the order $\mathrm{CD}>>\mathrm{MS}$ $>$ LD $>$ control $>$ HT $>$ FILA $=$ CC. The observed rates of superoxide production ranged from 2.58-14.4 nmol. $\mathrm{min}^{-1} /$ $\mathrm{mg}^{-1}$ a fivefold variation, while control mitochondria consistently showed values in the region of $5 \mathrm{nmoles}$. $\mathrm{min}^{-1} / \mathrm{mg}^{-1}$. This suggests that the rate of electron diversion to measurable superoxide production in the respiratory chain, estimated by dividing superoxide production rate by the activity of complex I + III, is of the order of $4 \%$ in control cell lines and may be as high as $33 \%$ in complex I deficiency as demonstrated by cell line 7123 (Table I).

The production of superoxide from the respiratory chain as a by-product of electron transport is believed to occur in proximity to sites of semiquinone production within complexes I and III (3). Since there are probably two sites within complex I and at least one in complex III where this could happen $(3,16)$ it is not surprising that excessive superoxide production occurs in defects of electron transport. It is, however, interesting that simple blockage of electron transport in complex I or III by the respiratory inhibitors rotenone or antimycin, respectively, increases the production of superoxide but not to the same extent as observed in CD (3). In our assay system, rotenone as an inhibitor of complex I increases the superoxide production rate by some $20 \%$ in both control and deficient cell lines, while antimycin A which inhibits complex III increased superoxide production by up to $80 \%$. Since rotenone is believed to act by binding to the ND1 protein situated spatially at the end of complex I $(17,22)$ in competition with either the oxidized or reduced form of CoQ10 (21), this might imply that increased levels of semiquinone generated in this vicinity might actually be the source of electrons for the reduction of molecular oxygen to superoxide. The alternative explanation is that defects in complex I present in this population predispose the respiratory chain to produce excess superoxide because spatial, structural, or stoichiometric alterations in the subunits within the complex itself allow increased interaction of $\mathrm{O}_{2}$ with an electron carrier. In any event, we must draw the conclusion that the increase in superoxide production seen in complex I deficiency cannot be explained simply as a response arising from blockage of electron transport. Mitochondria from cell lines with combined PDH complex, complex III, or complex IV deficiency do not consistently display increased superoxide production or increase of MnSOD activity despite severe restriction of the rate of electron transport. Two patients with complex IV deficiency and Leigh's disease did show significantly elevated MnSOD levels in mitochondria. This may indicate that a more detailed analysis of cell lines with complex IV deficiency is warranted.

The observed rate of superoxide production in mitochondrial preparations seemed to be highly dependent on the state of MnSOD induction. In fibroblast lines which showed high levels of MnSOD (CC, FILA, and HT) superoxide production rates were comparable with or depressed below control values. In those lines with normal or low levels of MnSOD (MS, $\mathrm{CD}, \mathrm{LD}$ ) superoxide production was increased (Fig. 7). Thus, 


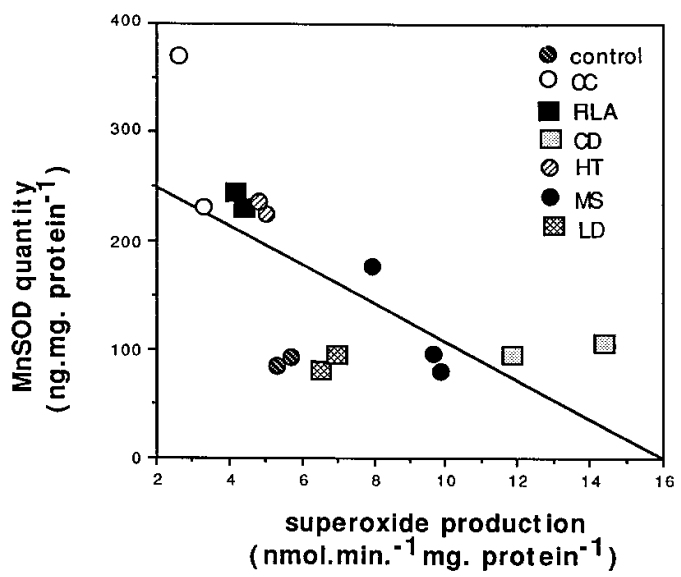

Figure 7. Plots of MnSOD quantity vs superoxide production showed an inverse relationship for most conditions with complex I deficiency. Superoxide production (steady state at $30 \mathrm{~s}$ value obtained from production curve; see Fig. 5) seemed to be highly dependent on the state of MnSOD induction. In fibroblast lines which showed high levels of MnSOD (CC, FILA, and HT) superoxide production rates were depressed below control values and those lines with normal or low levels of MnSOD ( $M S, C D$, and $L D$ ) superoxide production was the same as control or increased.

the actual observable superoxide production was dependent on the resultant dynamic between excessive radical production resulting from complex I deficiency and the response of the mitochondrial defence system designed to remove free radicals.

What then determines whether or not MnSOD is induced in the circumstances of a complex I defect and does this have any bearing on the severity of disease? The majority of research into MnSOD induction has been focused on the mechanisms involved in its induction in hyperoxia and by cytokines $(4,5)$. In the former case there seems to be a redox-sensitive mechanism involving MnSOD gene transcription mediated by an inducer protein which binds the upstream elements of the MnSOD gene (6). An alternative or adjunct process could involve a preregulatory protein which binds the $3^{\prime}$ end of the MnSOD mRNA and regulates translation (20). In the case of cytokine induction there is strong evidence from phorbol ester induction of MnSOD that the signaling pathway involves a protein kinase c-dependent mechanism (4). Here we have presented evidence that the induction of MnSOD is probably related to the extent of the redox abnormality ( $\mathrm{L} / \mathrm{P}$ ratio) in the cell produced by complex I deficiency and this would imply that the favored mechanism would involve a redox-sensitive mediator. Since we showed that increased MnSOD mRNA levels are present in complex I deficient cells with MnSOD induction, such a mechanism involving a redox regulated transcription factor would be consistent with the observations. The mechanism, however, must involve more than a simple change in redox state since MnSOD is only increased by $30 \%$ when control cells are cultured in the presence of rotenone (Table I). We propose that the significant induction of MnSOD in certain cases of complex I deficiency may therefore involve a temporarily much elevated superoxide production rate in the presence of an abnormally reduced redox state, i.e., the conditions that are very similar to those encountered in anoxia reperfusion injury.

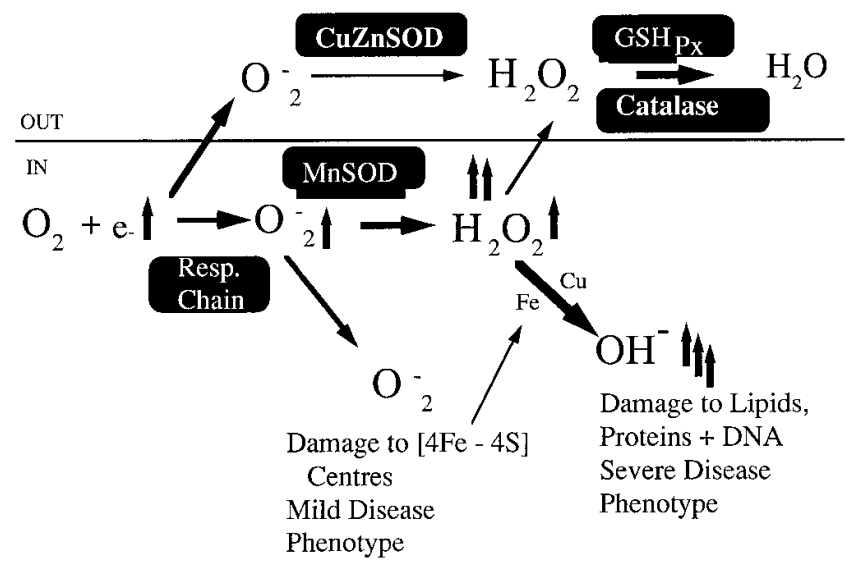

Figure 8. Scheme showing the major elements involved in the production and disposal of oxygen free radicals generated by the mitochondrial respiratory chain. Superoxide is produced by complexes I and III during electron transport inside the mitochondria. This superoxide can be removed to hydrogen peroxide by MnSOD or if produced outside mitochondria by $\mathrm{CuZnSOD}$. The hydrogen peroxide can be removed by catalase or glutathione peroxidase or it may react with superoxide in the presence of free $\mathrm{Cu}$ or $\mathrm{Fe}$ ions to produce the damaging hydroxyl radical. The severity of disease that develops in complex I deficiency may be influenced by the interactions of these pathways.

The patients with the worst prognosis (FILA, CC, and HT) were those with the highest induction of MnSOD while those with poor induction survived better (LD and MS) even when superoxide production itself was greatly elevated (CD). If the major determinant of superoxide production in complex I deficiency is the amount of induced MnSOD in mitochondria (20), and then mitochondria with high levels of MnSOD production are likely to develop the highest levels of hydrogen peroxide. If there was no MnSOD induction, for example, in the cases of cardiomyopathy with cataracts (CC), no doubt the observed rate of superoxide production would have been as high as that seen in the cases of complex I deficiency and cataracts (CD). It has been shown that superoxide specifically attacks (4Fe-4S) centers such as are present in complex I and II and aconitate hydratase and this results in release of free iron in mitochondria and cytosol $(19,20)$. In the presence of such high free iron levels hydrogen peroxide, the product of the MnSOD reaction, would generate excessive hydroxyl radicals in these cells by the Fenton reaction (3) (Fig. 8). The hydroxyl radical is most reactive of free oxygen radicals causing damage to DNA, lipids, and proteins (18), and would be likely to contribute to the severe symptoms, morbidity, and mortality observed in the patients where its generation is increased. In future experiments we will endeavor to examine hydroxyl radical production in order to determine whether these secondary free radicals are elevated selectively in certain patients with complex I deficiency.

\section{Acknowledgments}

We thank the Networks of Centres of Excellence Programme of the Canadian Federal Government, the National Foundation, the March of Dimes, the Emil Aaltonen Foundation, and the Pediatric Research Foundation of Finland for support. 


\section{References}

1. Boveris, A., N. Oshino, and B. Chance. 1972. The cellular production of hydrogen peroxide. Biochem. J. 128:617-630.

2. Takashige, K., and S. Minakami. 1979. NADH- and NADPH-dependent formation of superoxide anions by bovine heart submitochondrial particles and NADH-ubiquinone reductase preparation. Biochem. J. 180:129-135.

3. Beyer, R.E. 1992. An analysis of the role of coenzyme Q in free radical generation and as an oxidant. Biochem. Cell Biol. 70:390-403.

4. Hennet, T., C. Richter, and E. Peterhans. 1993. Tumor necrosis factoralpha induces superoxide anion generation in mitochondria of L929 cells. Biochem. J. 289:587-592.

5. Shull, S., N.H. Heintz, M. Periasamy, M. Manohar, Y.M.W. Janssen, J.P. Marsh, and B.T. Mossman. 1991. Differential regulation of antioxidant enzymes in response to oxidants. J. Biol. Chem. 266:24398-24403.

6. Pitkänen, S., F. Merante, D.R. McLeod, D. Applegarth, T. Tong, and B.H. Robinson. 1995. Familial cardiomyopathy and cataracts and lactic acidosis: A defect in complex I of the respiratory chain. Pediatr. Res. 39:513-521.

7. Robinson, B.H., N. MacKay, P. Goodyer, and G. Lancaster. 1985. Defective intramitochondrial NADH oxidation in skin fibroblasts from an infant with fatal neonatal lacticacidemia. Am. J. Hum. Genet. 37:938-946.

8. Robinson, B.H. D.M. Glerum, W. Chow, R. Petrova-Benedict, R Lightowlers, and R. Capaldi. 1990. The use of skin fibroblast cultures in the detection of respiratory chain defects in patients with lacticacidemia. Pediatr. Res. 28:549555.

9. Moreadith, R.W., M.L. Batshaw, T. Ohnishi, D. Kerr, B. Knowx, D. Jackson, R. Hruba, J. Olson, B. Reynafarje, and A.L. Lehninger. 1984. Deficiency of the iron-sulfur clusters of mitochondrial reduced nicotinamide-adenine dinucleotide-ubiquinone oxidoreductase (complex I) in an infant with congenital lactic acidoses. J. Clin. Invest. 74:685-697.

10. Di Mauro, S., J.F. Nicholson, A.P. Hays, A.B. Eastwood, A. Papadimitriou, R. Koeningsberger, and D.C. De Vivo. 1983. Benign infantile mitochondrial myopathy due to reversible cytochrome $c$ oxidase deficiency. Ann. Neurol. 14:226-234.
11. Fischer, J.C., W. Ruitenbeek, A.M. Stadhouders, J.M.F. Trijbels, R.C.A. Sengers, A.J.M. Janssen, and J.H. Veerkamp. 1985. Investigation of mitochondria metabolism in small human skeletal muscle biopsy specimens. Improvement of preparation procedure. Clin. Chim. Acta. 145:89-94.

12. Bhuyan, D.K., and K.C. Bhuyan. 1994. Assessment of oxidative stress to eye in animal model for cataract. Methods Enzymol. 233:630-639.

13. Betts, W.H. 1986. Detecting radicals by chemiluminescence. In Handbook of methods for oxygen radical research. CRC Press, Inc. Boca Raton, FL. 197-201.

14. Faulkner, K., and I. Fridovich. 1993. Luminol and lucigenin as detectors for $\mathrm{O}_{2}-$. Free Rad. Biol. Med. 15:447-451.

15. Glerum, M., W. Yanamura, R. Capaldi, and B.H. Robinson. 1988. Characterization of cytochrome oxidase mutants in human fibroblasts. FEBS Lett. 236:100-104.

16. Robinson, B.H. 1993. Lacticacidemia. Biochim. Biophys. Acta. 1182 231-244.

17. Earley, F.G.P., S.D. Patel, C.I. Ragan, and G. Attardi. 1984. Photolabeling of a mitochondrially encoded subunit of NADH dehydrogenase with $\left[{ }^{3} \mathrm{H}\right]$ dihydrorotenone. FEBS Lett. 219:108-113.

18. Fridovitch, I. 1995. Superoxide radical and superoxide dismutases. Ann. Rev. Biochem. 64:97-112.

19. Flint, D.H., J.F. Tuminello, and M.H. Emptage. 1993. The inactivation of Fe-S cluster containing hydro-lyases by superoxide. J. Biol. Chem. 268: 22369-22376.

20. Fazzone, H.A. Wagner, and L.B. Clerch. 1993. Rat lung contains a developmentally regulated manganese superoxide dismutase mRNA binding protein. J. Clin. Invest. 92:1278-1281.

21. Ueno, H., H. Miyoshi, K. Ebisui, and H. Iwamura. 1994. Comparison of the inhibitory action of natural rotenone and its stereoisomers with various NADH-ubiquinone reductases. Eur. J. Biochem. 225:411-417.

22. Yagi, T., and Y. Hatefi. 1988. Identification of the dicyclohexylcarbodiimide-binding subunit of NADH-ubiquinone oxidoreductase (complex I). $J$. Biol. Chem. 263:16150-16155. 